\title{
Self-Guided Supramolecular Cargo-Loaded Nanomotors with Chemotactic Behavior towards Cells
}

\author{
Fei Peng, Yingfeng Tu, Jan C. M. van Hest,* and Daniela A. Wilson*
}

\begin{abstract}
Delivery vehicles that are able to actively seek and precisely locate targeted tissues using concentration gradients of signaling molecules have hardly been explored. The directed movement toward specific cell types of cargo-loaded polymeric nanomotors along a hydrogen peroxide concentration gradient (chemotaxis) is reported. Through self-assembly, bowl-shaped poly(ethylene glycol)-b-polystyrene nanomotors, or stomatocytes, were formed with platinum nanoparticles entrapped in the cavity while a model drug was encapsulated in the inner compartment. Directional movement of the stomatocytes in the presence of a fuel gradient (chemotaxis) was first demonstrated in both static and dynamic systems using glass channels and a microfluidic flow. The highly efficient response of these motors was subsequently shown by their directional and autonomous movement towards hydrogen peroxide secreting neutrophil cells.
\end{abstract}

Chemotaxis is the process by which bacteria and immune cells navigate along a concentration gradient towards chemical attractants or away from repellants. ${ }^{[1]}$ This natural phenomenon has inspired scientists to construct synthetic motors able to demonstrate a similar behavior in vitro. Sen's group discovered that bimetallic rods and even single enzymes could show chemotactic behavior and move autonomously towards their substrates. ${ }^{[2]}$ Schmidt, Sanchez, and co-workers reported that tubular microjets and Janus motors showed a deviation towards areas with a higher concentration of hydrogen peroxide.$^{[3]}$ Chattopadhyay and co-workers described hydrogen peroxide-driven catalytic microspheres that were guided towards alkali-rich regions. ${ }^{[4]}$ These motors with chemotactic behavior offer the possibility, ${ }^{[5]}$ besides application of external magnetic ${ }^{[6]}$ or electric fields, ${ }^{[7]}$ to achieve precise directional control, ${ }^{[8]}$ which is still lacking in most artificial motors. However, most current chemotactic motors suffer from limited cargo loading potential and their size dimensions limit possible applications, for example with regard to drug delivery.

[*] F. Peng, Y. Tu, Dr. J. C. M. van Hest, Dr. D. A. Wilson Institute for Molecules and Materials, Radboud University Heyendaalseweg 135, 6525 AJ, Nijmegen (The Netherlands) E-mail: j.vanhest@science.ru.nl d.wilson@science.ru.nl

(6) Supporting information for this article is available on the WWW under http://dx.doi.org/10.1002/anie.201504186.

of (c) 2015 The Authors. Published by Wiley-VCH Verlag GmbH \& Co. $\mathrm{KGaA}$. This is an open access article under the terms of the Creative Commons Attribution Non-Commercial NoDerivs License, which permits use and distribution in any medium, provided the original work is properly cited, the use is non-commercial and no modifications or adaptations are made.
Several approaches have been taken towards developing biomedical applications of nanomotors. ${ }^{[9]}$ Nanosized selfassembled motors that can be loaded with cargo and also show chemotactic behavior have however not been demonstrated before. An interesting biologically relevant chemical signaling compound for induction of chemotaxis is hydrogen peroxide ${ }^{[10]}$ Compared to normal tissue, hydrogen peroxide is overproduced by tumor cells and facilitates cancer cell invasion and metastasis. ${ }^{[11]}$ A drug-delivery system that could autonomously move in interstitial tissue, seek out the hydrogen peroxide source, and deliver the antitumor agent would be a great improvement to current passively targeted drug delivery systems.

Herein we present a nanomotor that meets the requirements regarding size, loading capability and directional movement. ${ }^{[3,8,9]}$ The nanomotor is based on a polymersome stomatocyte functionalized with platinum nanoparticles (PtNP) and loaded with a model anticancer drug, doxorubicin (Dox) as cargo. Furthermore, we demonstrate that these nanomotors show motion towards higher concentrations of hydrogen peroxide, both in artificially created chemical gradients in a microfluidic device, as well as in the presence of hydrogen peroxide excreting neutrophils.

Through self-assembly, ${ }^{[12]}$ Dox and PtNP loaded stomatocytes with an average size of $308 \mathrm{~nm}$ were obtained (Supporting Information, Table S1). Results from transmission electron microscopy (TEM) (Supporting Information, Figure S1 a,b), cryogenic transmission electron microscopy (cryo TEM; Figure 1b) and 3D reconstruction (Figure 1c-e; Supporting Information, video S1) indicated that the vesicles have a bowl-shaped morphology with the PtNP entrapped in the outer cavity while confocal laser scanning microscopy (CLSM) (Figure 1i) and energy-dispersive X-ray spectroscopy $($ EDX) (Figure $1 \mathrm{~h}$ ) corroborate the successful incorporation of doxorubicin. (For Dox and PtNP encapsulation efficiency, PtNP - 59.8\%, Dox - 78.1\% see Supplementary Information.)

Once the structure of the nanomotor was confirmed to have incorporated both the engine (PtNP) and the cargo (Dox), we subsequently analyzed the speed of the nanomotors by using nanoparticle tracking analysis. Upon applying hydrogen peroxide, the PtNP containing stomatocytes showed a fuel concentration-dependent diffusion enhancement (Supporting Information, Figures S3, S4). The diffusion coefficient of PtNP stomatocytes experienced a remarkable increase from $1.74 \times 10^{-8} \mathrm{~cm}^{2} \mathrm{~s}^{-1}$ in water to $2.68 \times 10^{-8} \mathrm{~cm}^{2} \mathrm{~s}^{-1}$ upon addition of $5 \%(\mathrm{v} / \mathrm{v})$ of hydrogen peroxide (final concentration was $0.15 \% \mathrm{v} / \mathrm{v})$.

To evaluate the chemotatic behavior of our Dox and PtNP loaded stomatocytes, a glass flow device that is similar to a PP 

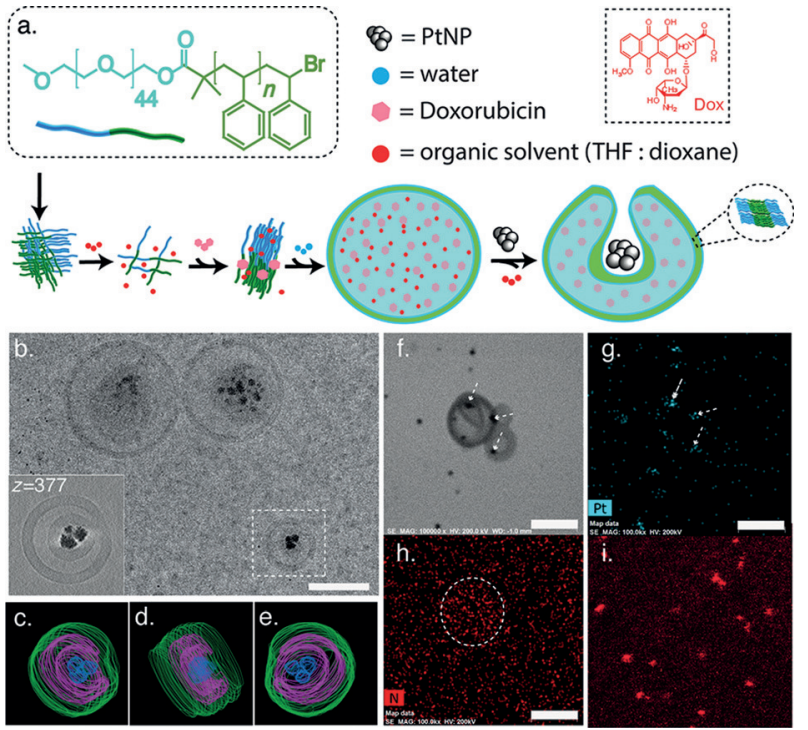

Figure 1. a) Procedure for preparing Doxorubicin (Dox) and platinum nanoparticle (PtNP) loaded stomatocytes. b) cryo-TEM images of Dox and PtNP loaded stomatocytes. c)-e) 3D reconstructed images of the loaded vesicle (dotted white line square in b) from cryo-TEM tomography at different angles. f) TEM images and EDX g) Pt h) N) mapping of Dox and PtNP loaded stomatocytes. All scale bars are $300 \mathrm{~nm}$. i) confocal images of PtNP and fluorescent Dox-loaded stomatocytes. a.
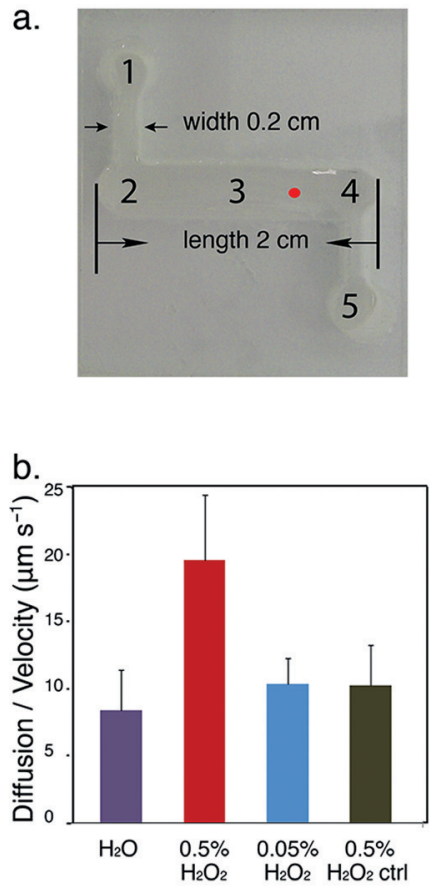

C. $\mathrm{H}_{2} \mathrm{O}$

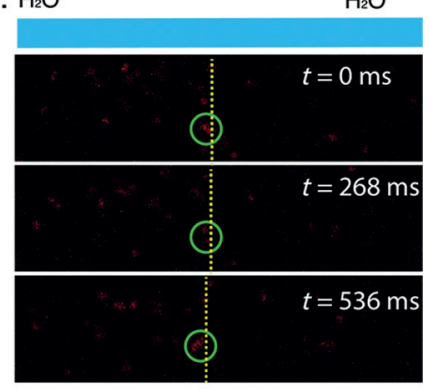

$\mathrm{H}_{2} \mathrm{O}_{2}$

d

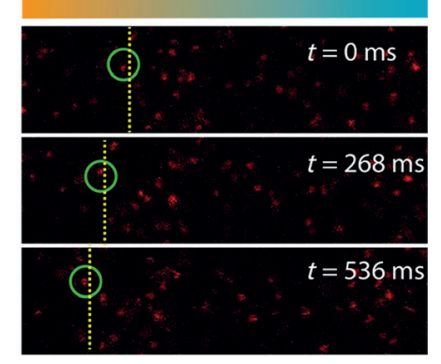

Figure 2. Chemotaxis evaluation in a glass channel: a) the glass channel design, b) Diffusion (for controls) or average velocity of Dox and PtNP loaded stomatocytes upon addition of $\mathrm{H}_{2} \mathrm{O}$ (control), $0.5 \% \mathrm{v} / \mathrm{v}_{2} \mathrm{O}_{2}$, and $0.05 \% \mathrm{v} / \mathrm{v} \mathrm{H}_{2} \mathrm{O}_{2}$ at position 1, or Dox loaded stomatocytes upon addition of $0.5 \% \mathrm{H}_{2} \mathrm{O}_{2}$ (control) after tracking 30 nanoparticles for each group.

c) Motion of Dox and PtNP loaded stomatocytes upon addition of $\mathrm{H}_{2} \mathrm{O}$ or

(d) $0.5 \% \mathrm{H}_{2} \mathrm{O}_{2}$. In the green circles the movement of a single particle is tracked. Scale bars: $4 \mu \mathrm{m}$. chamber $^{[13]}$ for cell chemotaxis evaluation was designed (Figure 2a) and confocal fluorescence microscopy was employed. The design of the channel, including the size and shape, was chosen to minimize possible drift and convection effects caused by the addition of the hydrogen peroxide/ stomatocytes solution. The hydrogen peroxide concentration gradient was first demonstrated by using a hydrogen peroxide sensitive dye 2,7-dichlorodihydrofluorescein from which an optimal observation region (red spot between position 3 and 4, Figure $2 \mathrm{a}$ ) was determined (see Supporting Information). In the experiment group, $5 \mu \mathrm{L}$ of freshly prepared Dox and PtNP loaded stomatocytes was added to position 5 after the glass channel was filled with deionized water, and $5 \mu \mathrm{L}$ of a hydrogen peroxide solution was added to position 1 to generate a concentration gradient (Figure S5). In case of the $0.5 \% \mathrm{H}_{2} \mathrm{O}_{2}$ experiment it was observed at the red spot position (Figure $2 \mathrm{a}$ ) that tracked particles (overlaid with green circles) showed a net movement to the left where the hydrogen peroxide levels were higher (Figure 2d). Similar directional movement was also observed for Dox and PtNP loaded stomatocytes in the presence of $0.05 \%$ of hydrogen peroxide, at a lower velocity (Figure $2 b$ ).

An increased movement rate along the hydrogen peroxide concentration gradient was also observed. Motors at position 4 with an average velocity of $15.47 \mu \mathrm{m} \mathrm{s}^{-1}$ accelerated to $19.64 \mu \mathrm{m} \mathrm{s}^{-1}$ at the red spot position with addition of $0.5 \%$ hydrogen peroxide, while motors with $0.05 \%$ hydrogen peroxide addition experienced a slight increase from $10.06 \mu \mathrm{m} \mathrm{s}^{-1}$ to $10.33 \mu \mathrm{m} \mathrm{s}^{-1}$ from position 4 to the red spot, consistent with the results from the nanosight experiment (Supporting Information, Figure S3). When moving up the gradient, motors travelled longer distances than by moving in the opposite direction, resulting in net motion towards the hydrogen peroxide rich area, which accounts for their chemotactic behavior. Control experiments were further performed and possible influences of the Marangoni effect, ${ }^{[14]}$ drift effects, channel wall interaction, ${ }^{[15]}$ and convection gradients ${ }^{[16]}$ were ruled out (Supporting Information). The net displacement of the nanomotors was also confirmed by observations with both microscopy and the naked eye. As the cluster of Pt-loaded nanomotor appeared dark, their displacement could be directly observed. The lack of movement in the negative control samples explains the reason that fewer particles were observed in Figure $2 \mathrm{c}$ than in Figure $2 \mathrm{~d}$.

To further demonstrate the chemotactic behavior of Dox and PtNP loaded stomatocytes, a microfluidic twoinlet one-outlet flow device was used (Figure 3, left). The design of the device offers tuneable control over the fluid flow and concentration gradient generation. To establish a hydrogen peroxide concentration gradient with the least possible diffusion, the optimal flow rate through each inlet was investigated and determined to be $600 \mu \mathrm{Lh}^{-1}$, which was maintained constant throughout the whole flow device experiment (Supporting Information). Dox and PtNP loaded stomatocytes were pumped into the device through inlet 2 while hydrogen peroxide of $0.05 \% \mathrm{v} / \mathrm{v}$ was injected through inlet 1 . Hydrogen 


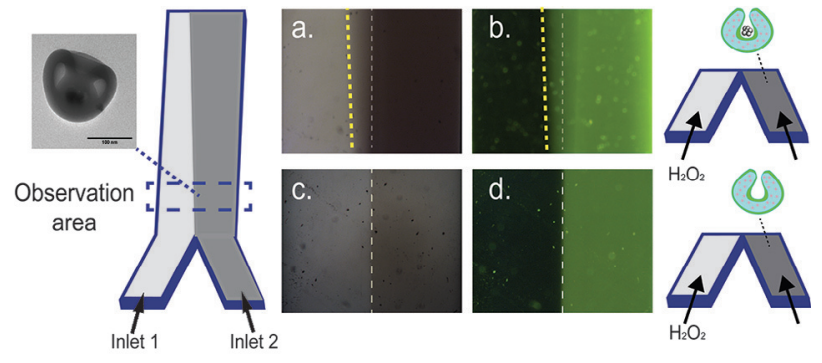

Figure 3. Chemotaxis evaluation in a 3D microfluidic channel. Left: Representation of the microfluidic channel. Lateral shifts of Dox and PtNP loaded stomatocytes in the presence of $0.05 \% \mathrm{H}_{2} \mathrm{O}_{2}$ : bright field (a) and fluorescent imaging (b), No deviation towards the fuel of the only Dox loaded stomatocytes in presence of $0.05 \% \mathrm{H}_{2} \mathrm{O}_{2}$ : bright field (c) and fluorescent imaging (d). Scale bar: $100 \mathrm{~nm}$.

peroxide with a relatively low concentration of $0.05 \%$ was chosen so as to make sure no visible oxygen bubbles would be produced initially and thereby perturb the flow profile. The particle concentration was held constant during the flow experiments.

Measurements started after a steady-state profile was obtained. The motors exhibited a lateral shift towards channel1 (Figure 3a,b) while in the case of water instead of hydrogen peroxide only straight motion was observed and the deviation angle was close to zero (Supporting Information, Figure S9a,b). It is possible that convection may cause bulk fluid distortion. To exclude the flow effects, control experiments with Dox only-loaded stomatocytes were performed. No deviation towards the hydrogen peroxide containing channel was observed in this case (Figure $3 \mathrm{c}, \mathrm{d}$ ). To further rule out the possible influence of channel asymmetry or channel flow differences on the flow profile, Dox and PtNP containing stomatocytes were injected into inlet 1 and hydrogen peroxide through inlet 2. A lateral shift towards inlet2 was observed for Dox and PtNP loaded stomatocytes (Supporting Information, Figure $\mathrm{S} 9 \mathrm{c}, \mathrm{d}$ ) confirming that they move towards higher concentrations of hydrogen peroxide irrespective of the channel used.

After establishing the chemotactic behavior of these nanomotors, we set out to investigate if they could also respond to hydrogen peroxide gradients provided by model cells. This would provide the proof of concept of active delivery vehicles able to guide their movement towards diseased cells. We explored this possibility by using activated neutrophils as a hydrogen peroxide source ${ }^{[17]}$ Neutrophils were isolated from freshly heparinized rabbit blood. ${ }^{[18]}$ The survival rate and neutrophil density of the obtained preparation was determined to be $90.9 \%$ and $1.8 \times 10^{8}$ cells $\mathrm{mL}^{-1}$, respectively, using trypan blue staining and hemocytometer counting. Then phorbol 12-myristate 13acetate activated neutrophils were seeded into a HBSS soaked cell culture substrate before being placed into the center of a petri dish filled with Dox and PtNP loaded stomatocytes. At an observation time of $1.5 \mathrm{~h}$, the nanomotors were found to be translating towards the activated neutrophils. As a control, we placed only HBSS soaked substrate into the nanomotor-filled petri dish. No such phenomenon was observed in this case. As the substrate was immersed in the HBSS solution for 30 min and was kept inside the solution during the experiment, no capillary forces ${ }^{[19]}$ between the substrate and air were expected to cause any directional motion of the nanomotors. Furthermore a lack of directional motion was also observed when activated neutrophils were placed into a solution of Dox-only loaded stomatocytes. After normalizing the starting point of a number of stomatocytes to zero, the trajectories of particles over fine consecutive frames (total time interval=1.34 s) from three groups could be compared. The only group with active neutrophils and nanomotors showed directional movement (Figure $4 \mathrm{~d}$ ). The tracking paths of nanoparticles at position 1 are shown in Figure $4 \mathrm{~d}$. Figure $4 \mathrm{~d}-\mathrm{f}$ are the tracking paths of nanoparticles at position 1. For the nanomotors with activated cells group, the motion at position 2 and 3 is less directional than at position 1 (Figure $4 \mathrm{~d}$ ). The distance between the center of mass or the spatial average end point and the point of origin
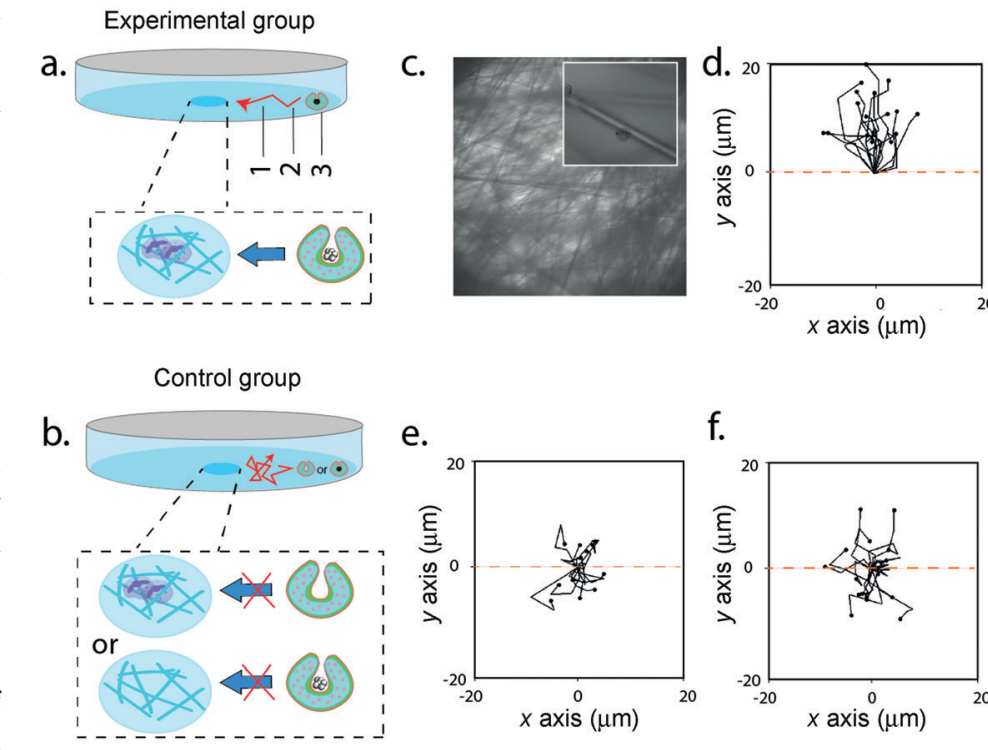

f.
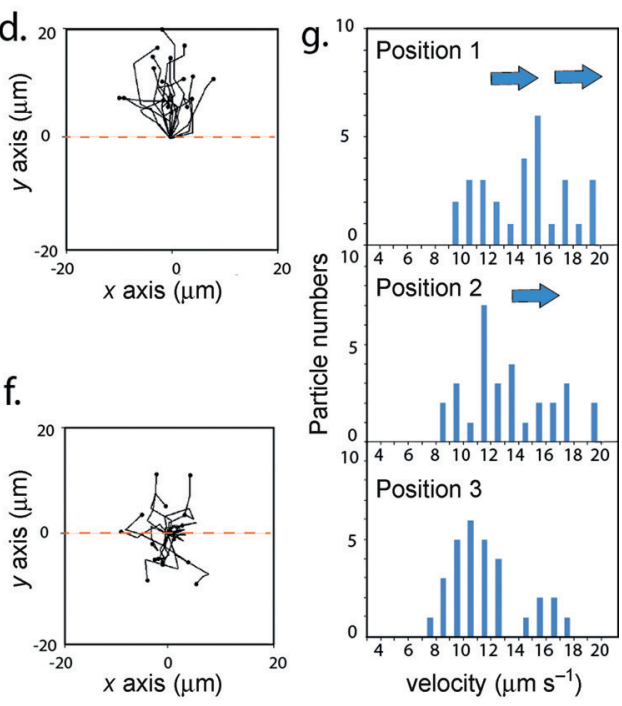

Figure 4. a) Experiment group set up for chemotaxis evaluation with neutrophils as hydrogen peroxide source and Dox-PtNP loaded stomatocytes in the solution. b) Control group set up using either Dox-PtNP loaded stomatocytes without cells, or neutrophils with Dox-only loaded stomatocytes. c) Bright field microscopy image of the center of the petri dish with the cells immobilized onto the cell culture substrate. The activated neutrophils can be observed at higher magnification in the inset. d) Tracking paths over 5 consecutive frames of the Dox-PtNP loaded nanomotors moving directionally towards the activated neutrophils, e) PtNP loaded nanomotors without activated neutrophils, f) Dox-only loaded stomatocytes with activated neutrophils, at position 1. g) Velocity distribution at position 1, 2, 3 for the experiment group. 
(displacement of center of mass) were also compared. As the nanomotors showed a preferred migration and directional movement in the presence of activated neutrophils, the displacement value of the center of mass was higher than the displacement values of the control group, which was close to zero (Table S2). It was also shown that the velocity of the nanomotors gradually increased as they were moving closer to substrate, varying from an average velocity of $12.4 \mu \mathrm{m} \mathrm{s}^{-1}$ at position 3 to an average velocity of $15.6 \mu \mathrm{m} \mathrm{s}^{-1}$ at position 1 (Figure $4 \mathrm{~g}$ ). We assume the directional motion is attributed to an enhanced diffusion of motors at a higher concentration of hydrogen peroxide. The multimodality of the velocity distribution could be due to the loading difference of catalytic PtNP between vesicles as observed with cryo-TEM (Figure $1 b)$.

In conclusion, we have demonstrated that nanosized soft polymer stomatocytes, loaded with platinum nanoparticles and the model drug doxorubicin, display chemotactic behavior in the presence of hydrogen peroxide gradients. Furthermore these structures can also sense hydrogen peroxideproducing cells by guiding their motion towards activated neutrophils. Chemotactic behavior can be explained by the fact that the nanomotors travel longer distances in the presence of higher concentration of fuels as their speed increases with fuel concentration, than in the opposite direction, leading to motors moving up the fuel gradient. While recent theoretical calculations point out that the extent of enhanced tumbling/rotating motion at higher fuel zone determines the chemotaxis direction (positive/negative) of motors, ${ }^{[20]}$ the exact chemotaxis mechanism remains to be an open question.

The nanosized dimensions of the motors and their ability to transport cargo make them an interesting starting point for the development of drug carrier systems with chemotactic potential.

Nanomotors with chemotactic behavior which could move against flow may find application in delivering drugs to latestage tumors where high interstitial pressure ${ }^{[21]}$ inhibits entrance of current delivery agents. ${ }^{[22]}$

\section{Acknowledgements}

This work was supported by the European Research Council under the European Union's Seventh Framework Programme (FP7/2007-20012)/ERC-StG 307679 "StomaMotors". We acknowledge support from the Ministry of Education, Culture and Science (Gravitation program 024.001.035). F. Peng acknowledges funding from China scholarship council. We would like to thank Elisabeth Pierson, GeertJan Janssen from General Instruments from Radboud University Nijmegen and Pekka Kujala for assistance with the Cryo-TEM analysis and TEM-EDX experiments.

Keywords: active drug delivery $\cdot$ chemotaxis $\cdot$ nanomotors . self-assembly

How to cite: Angew. Chem. Int. Ed. 2015, 54, 11662-11665 Angew. Chem. 2015, 127, 11828-11831
[1] R. R. Kay, P. Langridge, D. Traynor, O. Hoeller, Nat. Rev. Mol. Cell Biol. 2008, 9, 455-463.

[2] a) Y. Hong, D. Velegol, N. Chaturvedi, A. Sen, Phys. Chem. Chem. Phys. 2010, 12, 1423-1435; b) S. Sengupta, K. K. Dey, H. S. Muddana, T. Tabouillot, M. E. Ibele, P. J. Butler, A. Sen, J. Am. Chem. Soc. 2013, 135, 1406-1414.

[3] L. Baraban, S. M. Harazim, S. Sanchez, O. G. Schmidt, Angew. Chem. Int. Ed. 2013, 52, 5552-5556; Angew. Chem. 2013, 125, $5662-5666$.

[4] K. K. Dey, S. Bhandari, D. Bandyopadhyay, S. Basu, A. Chattopadhyay, Small 2013, 9, 1916-1920.

[5] A. K. Singh, K. K. Dey, A. Chattopadhyay, T. K. Mandal, D. Bandyopadhyay, Nanoscale 2014, 6, 1398-1405.

[6] T. R. Kline, W. F. Paxton, T. E. Mallouk, A. Sen, Angew. Chem. Int. Ed. 2005, 44, 744-746; Angew. Chem. 2005, 117, 754-756.

[7] P. Calvo-Marzal, K. M. Manesh, D. Kagan, S. Balasubramanian, M. Cardona, G. U. Flechsig, J. Posner, J. Wang, Chem. Commun. 2009, 4509-4511.

[8] J. Wang, K. M. Manesh, Small 2010, 6, 338-345.

[9] a) Z. Wu, X. Lin, Y. Wu, T. Si, J. Sun, Q. He, ACS Nano 2014, 8, 6097-6105; b) Z. Wu, T. Li, J. Li, W. Gao, T. Xu, C. Christianson, W. Gao, M. Galarnyk, Q. He, L. Zhang, J. Wang, ACS Nano 2014, 8, 12041-12048; c) S. Sánchez, L. Soler, J. Katuri, Angew. Chem. Int. Ed. 2015, 54, 1414-1444; Angew. Chem. 2015, 127, 1432 -1464; d) J. Wang, W. Gao, ACS Nano 2012, 6, 5745-5751; e) M. Guix, C. C. Mayorga-Martinez, A. Merkoci, Chem. Rev. 2014, 114, 6285-6322.

[10] P. Niethammer, C. Grabher, A. T. Look, T. J. Mitchison, Nature 2009, 459, 996-999.

[11] a) W. A. Wlassoff, C. D. Albright, M. S. Sivashinski, A. Ivanova, J. G. Appelbaum, R. I. Salganik, J. Pharm. Pharmacol. 2007, 59, 1549-1553; b) T. P. Szatrowski, C. F. Nathan, Cancer Res. 1991, 51,794-798; c) U. E. Martinez-Outschoorn, Z. Lin, C. Trimmer, N. Flomenberg, C. Wang, S. Pavlides, R. G. Pestell, A. Howell, F. Sotgia, M. P. Lisanti, Cell cycle 2011, 10, 2504-2520.

[12] D. A. Wilson, R. J. Nolte, J. C. van Hest, Nat. Chem. 2012, 4, $268-274$

[13] L. Köhidai, Curr. Microbiol. 1995, 30, 251-253.

[14] H. Jin, A. Marmur, O. Ikkala, R. H. A. Ras, Chem. Sci. 2012, 3, $2526-2529$.

[15] G. Dunderdale, S. Ebbens, P. Fairclough, J. Howse, Langmuir 2012, 28, 10997-11006.

[16] A. R. Morgan, A. B. Dawson, H. S. Mckenzie, T. S. Skelhon, R. Beanland, H. P. W. Franks, S. A. F. Bon, Mater. Horiz. 2014, 1, $65-68$.

[17] a) M. Freitas, G. Porto, J. L. Lima, E. Fernandes, Clin. Biochem. 2008, 41, 570-575; b) E. S. Olson, J. Orozco, Z. Wu, C. D. Malone, B. Yi, W. Gao, M. Eghtedari, J. Wang, R. F. Mattrey, Biomaterials 2013, 34, 8918-8924; c) F. Dallegri, G. Frumento, F. Patrone, Immunology 1983, 48, 273-279.

[18] P. Eggleton, R. Gargan, D. Fisher, J. Immunol. Methods 1989, $121,105-113$.

[19] A. A. Solovev, Y. Mei, O. G. Schmidt, Adv. Mater. 2010, 22, $4340-4344$.

[20] S. Saha, R. Golestanian, S. Ramaswamy, Phys. Rev. E 2014, 89, 062316.

[21] a) Y. Fan, W. Du, B. He, F. Fu, L. Yuan, H. Wu, W. Dai, H. Zhang, X. Wang, J. Wang, X. Zhang, Q. Zhang, Biomaterials 2013, 34, 2277-2288; b) X. Wang, C. Yang, Y. Zhang, X. Zhen, W. Wu, X. Jiang, Biomaterials 2014, 35, 6439-6453.

[22] I. K. Kwon, S. C. Lee, B. Han, K. Park, J. Controlled Release 2012, 164, 108-114.

Received: May 12, 2015

Revised: July 9, 2015

Published online: August 14, 2015 